\title{
Breastfeeding and circulating immunological markers during the first 3 years of life: the DIABIMMUNE study
}

\author{
Maija E. Miettinen ${ }^{1}$ (D) $\cdot$ Jarno Honkanen ${ }^{2}$ - Sari Niinistö ${ }^{1}$ - Outi Vaarala ${ }^{2}$ Suvi M. Virtanen ${ }^{1,3,4,5} \cdot$ Mikael Knip $^{6,7,8,9}$. \\ the DIABIMMUNE Study Group
}

Received: 21 March 2021 / Accepted: 20 August 2021 / Published online: 27 November 2021

(C) The Author(s) 2021

\begin{abstract}
Aims/hypothesis Our aim was to study the association between duration of breastfeeding and circulating immunological markers during the first 3 years of life in children with HLA-conferred susceptibility to type 1 diabetes.

Methods We performed a longitudinal analysis of 38 circulating immunological markers (cytokines, chemokines and growth factors) in serum samples from Finnish (56 individuals, 147 samples), Estonian (56 individuals 148 samples) and Russian Karelian children (62 individuals, 149 samples) at 3, 6, 12, 18, 24 and 36 months of age. We also analysed gut inflammation markers (calprotectin and human $\beta$ defensin-2) at $3(n=96)$ and 6 months $(n=153)$ of age. Comparisons of immunological marker medians were performed between children who were breastfed for 6 months or longer vs children who were breastfed for less than 6 months.

Results Breastfeeding for 6 months or longer vs less than 6 months was associated with lower median of serum immunological markers at 6 months (granulocyte-macrophage colony-stimulating factor [GMCSF], macrophage inflammatory protein [MIP$3 \alpha]$ ), 12 months (IFN- $\alpha 2$, vascular endothelial growth factor, GMCSF, IFN- $\gamma$, IL-21), 18 months (FGF-2, IFN- $\alpha 2$ ) and 24 months of age (CCL11 [eotaxin], monocyte chemoattractant protein-1, TGF $\alpha$, soluble CD40 ligand, IL-13, IL-21, IL-5, MIP-1 $\alpha)$ (all $p<0.01$ ) but not at 36 months of age. Breastfeeding was not associated with gut inflammation markers at 3 and 6 months of age.

Conclusions/interpretation Children who were breastfed for 6 months or longer had lower medians for 14 immunological markers at one or more age points during the first 2 years of life compared with children who were breastfed for less than 6 months. The clinical meaning of the findings is not clear. However, the present study contributes to the understanding of immunological differences in children that have been breastfed longer, and thus provides a mechanistic suggestion for the previously observed associations between breastfeeding and risk of type 1 diabetes.
\end{abstract}

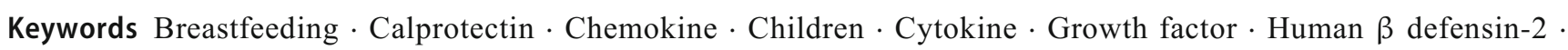
Immunological marker $\cdot$ Type 1 diabetes

Maija E. Miettinen

maija.miettinen@thl.fi

1 Department of Public Health and Welfare, Health and Well-Being Promotion Unit, Finnish Institute for Health and Welfare, Helsinki, Finland

2 Research Program for Clinical and Molecular Metabolism, Faculty of Medicine, University of Helsinki, Helsinki, Finland

3 Faculty of Social Sciences, Unit of Health Sciences, Tampere University, Tampere, Finland

4 Tampere University Hospital, Research, Development and Innovation Center, Tampere, Finland
5 Center for Child Health Research, Tampere University and Tampere University Hospital, Tampere, Finland

6 Children's Hospital, University of Helsinki and Helsinki University Hospital, Helsinki, Finland

7 Folkhälsan Research Center, Helsinki, Finland

8 Research Program for Clinical and Molecular Metabolism, University of Helsinki, Helsinki, Finland

9 Department of Pediatrics, Tampere University Hospital, Tampere, Finland 


\section{Research in context}

\section{What is already known about this subject?}

- Breastfeeding is associated with desirable colonisation of the infant gut

- Breastfeeding is associated with lower risk of type 1 diabetes

\section{What is the key question?}

- Is breastfeeding associated with circulating immunological markers and gut inflammation markers during the first 3 years of life?

\section{What are the new findings?}

- Breastfeeding for 6 months or longer compared with less than 6 months is associated with lower medians for 14 circulating immunological markers at one or more age points $(6,12,18,24$ months)

- Breastfeeding for 6 months or longer compared with less than 6 months is not associated with gut inflammation markers at 6 months of age

\section{How might this impact on clinical practice in the foreseeable future?}

- The study emphasises the importance of breastfeeding as one factor modulating the infant immune system

$\begin{array}{ll}\text { Abbreviations } \\ \text { CCL11 } & \begin{array}{l}\text { Eotaxin } \\ \text { GMCSF }\end{array} \\ & \begin{array}{l}\text { Granulocyte-macrophage colony-stimulating } \\ \text { factor }\end{array} \\ \text { MCP-1 } & \text { Monocyte chemoattractant protein-1 } \\ \text { MinDC } & \text { Minimum detectable concentration } \\ \text { MIP } & \text { Macrophage inflammatory protein } \\ \text { SCD40L } & \text { Soluble CD40 ligand } \\ \text { VEGF } & \text { Vascular endothelial growth factor }\end{array}$

\section{Introduction}

The prevalence of childhood-onset immune-mediated diseases, including type 1 diabetes, asthma and allergy, has considerably increased especially in high- and middle-income countries during the past decades. Epidemiological data suggest that early-life environmental exposures are key determinants of these diseases [1]. The so-called hygiene hypothesis has been suggested to explain the disease increase by diminishing early-life microbial and parasite infections, which may be needed to prevent harmful immune responses later in life, but underlying mechanisms are unclear [2].

Breastfeeding after birth has been reported to support the immature immune system of an infant through various immunomodulating components present in breast milk including anti-inflammatory cytokines. Breastfeeding is strongly associated with the development of gut microbiota, providing a desirable microbial colonisation of the gut, as demonstrated by higher levels of Bifidobacterium species in breastfed infants [3]. Additionally, breastfeeding has been associated with lower diversity and slower maturation of the gut microbiome [3].

Breastfeeding associates with lower concentration of serum and gut inflammation markers in infants after birth [4] but these associations have not been consistent or thoroughly investigated. Preterm infants have defective maturation of the immune system including lower production of various cytokines. Cytokines present in breast milk have been implicated in helping infants to form a sufficient immune response [5]. However, it is not known whether longer breastfeeding affects and possibly continues to benefit the developing immune system. Breastfeeding has been associated with lower risk of type 1 diabetes or islet autoimmunity in several studies [6], although the mechanism remains open to debate.

The aim of this study was to evaluate the association between breastfeeding and both circulating immunological markers and gut inflammation markers during the first 3 years of life.

\section{Methods}

Study population All new-born infants born between September 2008 and February 2011 in one hospital in Finland, two hospitals in Estonia and two hospitals in Russian Karelia were screened for HLA-conferred susceptibility to type 1 diabetes. Children with genotypes that increase the risk of the disease were invited to the birth cohort of the DIABIMMUNE study and followed prospectively from birth up to 3 years of age. From 835 children originally included in 
the study, 38 were excluded due to incomplete data, leaving 797 children (386 in Finland, 322 in Estonia, and 89 in Russia) [7]. Of the 797 children, analysis of circulating immunological markers were performed in children that had unthawed serum samples available (56 children and 147 samples from Finland, 56 children and 148 samples from Estonia and 62 children and 149 samples from Russian Karelia) from when children were 3, 6, 12, 18, 24 and 36 months of age. Gut inflammation markers (calprotectin and human $\beta$ defensin-2) were analysed in the $3(n=96)$ and 6 month $(n=153)$ samples. Breastfeeding status was recorded at each time point. The local ethics committees (Ethics committee, Helsinki and Uusimaa Hospital District; Ethics Review Committee on Human Research of the University of Tartu; and Ethics committee, Ministry of Health and Social Development, Karelian Republic of the Russian Federation) approved the study and parents provided written informed consents.

HLA genotyping The cord blood samples from the new-born infants were screened for HLA DR/DQ genotypes associated with increased risk for type 1 diabetes. Children positive for $D R 3-D Q 2$ (DQA1*05-DQB1*02) and/or DR4-DQ8 $(D R B 1 * 04: 01 / 2 / 4 / 5 / 8-D Q B 1 * 0302 / 4)$ without protective haplotypes were eligible for the study. Children carrying any of the following protective haplotypes were excluded: $D Q B 1 * 03: 01, D Q B 1 * 06: 02, D Q B 1 * 06: 03, D R B 1 * 04: 03$, $(D R 14)-D Q B 1 * 05: 03$ and (DR7)-DQA1*02:01$D Q B 1 * 03: 03$.

Serum immunological markers The concentrations of circulating cytokines, chemokines and growth factors were analysed from unthawed serum samples with Luminex technology using the 38-plexed Milliplex MAP Kit (cat. no. HCYTMAG-60K-PX38) according to the manufacturer's recommendations (Merck-Millipore Corp., Billerica, MA, USA). Analyses were performed with single reactions using undiluted serum samples. Quantification of the markers was performed with the Bio-Plex 200 Luminex instrument and Bio-Plex Manager software (Bio-Rad, Sweden). The concentration of each marker was determined from an eight-point standard curve using five-parameter logistic regression. The minimum detectable concentration (MinDC) was determined for each marker separately using the lowest concentration on the standard curve linear phase $(\mathrm{MinDC}=\mathrm{C}(\mathrm{low})+2 \mathrm{SD})$. The samples below the MinDC were given a value of $50 \%$ of MinDC.

Comparisons of immunological marker medians were performed between children who were breastfed for 6 months or longer vs children who were breastfed for less than 6 months. The numbers of children breastfed for less than 3 months or for 12 months or longer were low, thus preventing meaningful comparisons at the age of 3 or 12 months.
Statistical analyses Serum immunological marker and gut inflammation marker data are expressed as medians. Differences in serum and gut inflammation marker medians were compared using the Mann-Whitney $U$ test. $p$ values $<0.01$ were considered statistically significant. The analyses were performed using IBM SPSS Statistics for Windows, Version 27.0 (Released 2020; IBM Corp. Armonk, NY, USA).

\section{Results}

The mean duration of exclusive breastfeeding was 1.1 months in Finland, 1.4 months in Estonia and 3.3 months in Russian Karelia $(p<0.001)$. The total mean duration of breastfeeding was 9.1 months in Finland, 9.3 months in Estonia and 7.4 months in Russian Karelia $(p=0.046)$.

Breastfeeding for 6 months or longer compared with less than 6 months was associated with lower median of serum immunological markers at 6 months (granulocyte-macrophage colony-stimulating factor [GMCSF], macrophage inflammatory protein [MIP]-3 $\alpha$ ), 12 months (IFN- $\alpha 2$, vascular endothelial growth factor [VEGF], GMCSF, IFN- $\gamma$, IL21), 18 months (FGF-2, IFN- $\alpha 2$ ) and 24 months of age (eotaxin [CCL11], monocyte chemoattractant protein-1 [MCP-1], TGF- $\alpha$, soluble CD40 ligand [sCD40L], IL-13, IL-21, IL-5, MIP-1 $\alpha$ ) (all $p<0.01)$ (Table 1). Borderline association $(p<0.05)$ was found between breastfeeding for 6 months or longer with lower median of several serum immunological markers at $6,12,18$ and 24 months of age. No associations were found at 36 months of age.

Altogether, 78 and 116 children had both breastfeeding status and gut inflammation marker results available at 3 months of age and 6 months of age, respectively. Breastfeeding for 3 or 6 months or longer compared with less than 3 or 6 months was not associated with gut inflammation markers (human $\beta$ defensin-2 and calprotectin) at 3 or 6 months of age.

Altogether, nine children seroconverted to islet autoimmunity and one child developed type 1 diabetes. Given the low number of children with islet autoimmunity or type 1 diabetes and given the high individual variation of inflammation marker concentrations, meaningful analyses according to disease outcomes could unfortunately not be performed.

\section{Discussion}

We found associations between circulating immunological markers and breastfeeding at several time points during the first 24 months of life. These results provide novel information on the relationship between breastfeeding and the immune system during early childhood. 


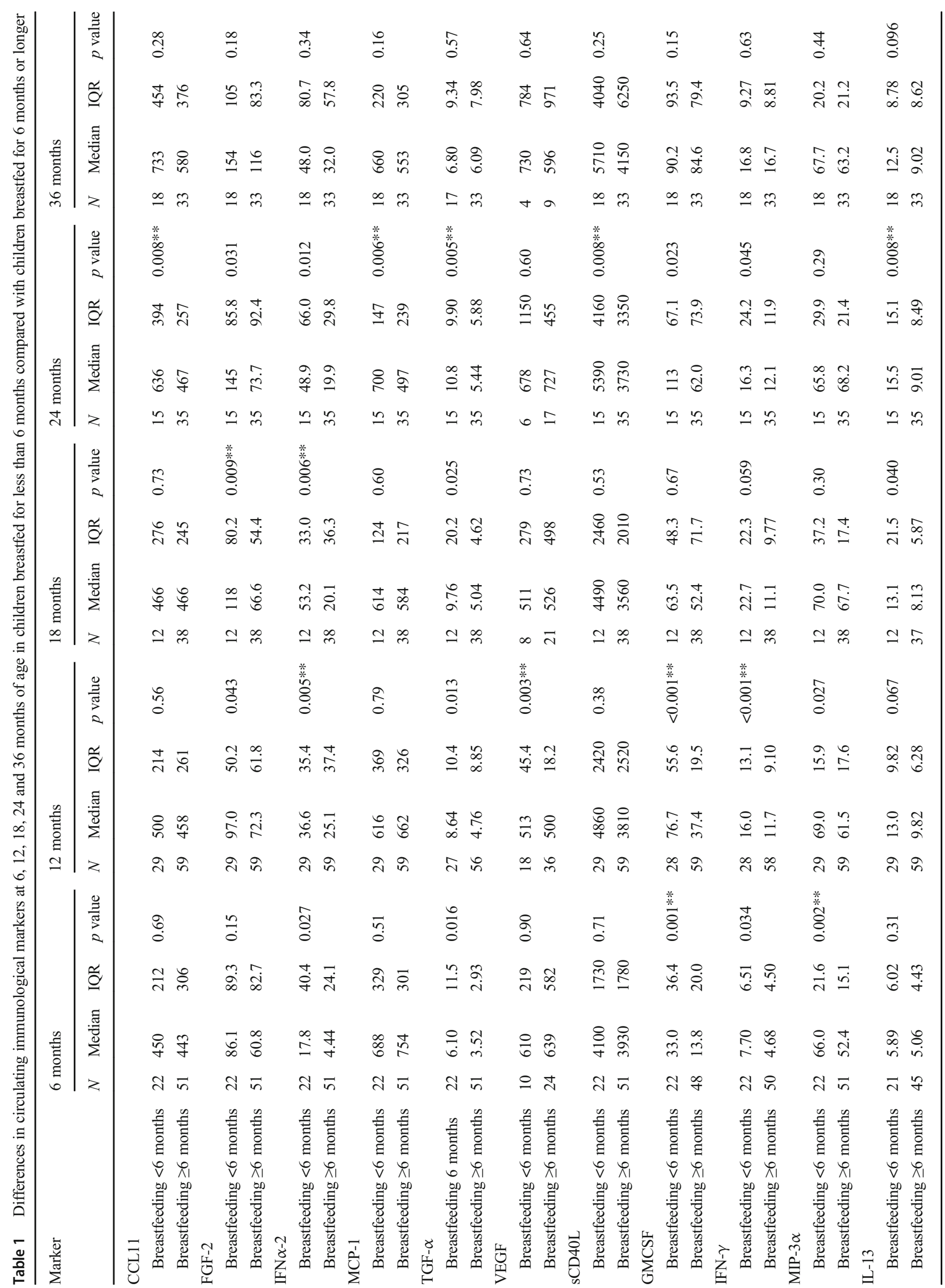




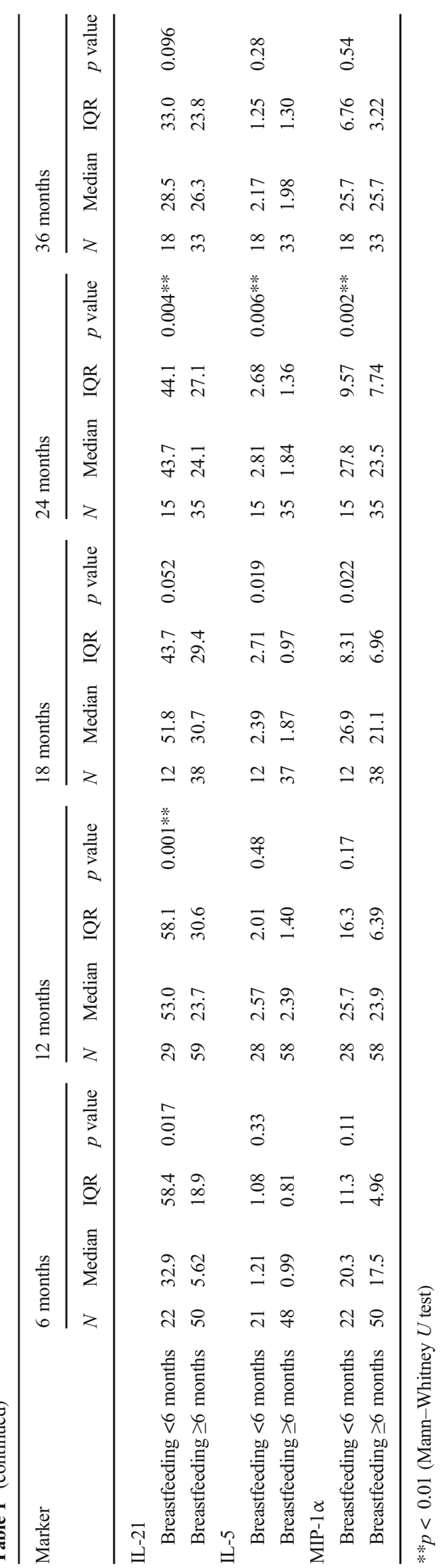


The strengths of the study include repeated measurements of a large array of circulating cytokines, chemokines and growth factors during the first years of life. Given the fact that the DIABIMMUNE study comprises children from Finland, Estonia and Russia, it would be interesting to determine whether the association between circulating immunological markers and breastfeeding would show differences within these three countries. The sample size was, however, so small in each individual country that meaningful analyses could not be performed. It was seen, however, that the duration of exclusive breastfeeding was higher in Russian Karelia than in Finland and Estonia, although the total duration of breastfeeding showed no large differences between the three countries.

IFN- $\alpha$ has been reported to be associated with several autoimmune diseases including type 1 diabetes. Increased expression of genes stimulated by INF- $\alpha$ have been seen in pancreatic biopsies taken from individuals with recent-onset type 1 diabetes compared with islets from control organ donors [8]. Both the Finnish DIPP [9] and the German BABYDIET study [10] reported that the IFN- $\alpha$ signature is temporally increased prior to the development of autoantibodies. In the present study, difference in IFN- $\alpha 2$ was seen at 12 and at 18 months of age, and also a nominal difference at 6 and at 24 months of age, strongly suggesting that breastfeeding modulates IFN- $\alpha 2$ production. More detailed analyses are needed, however, to understand the potential clinical significance of this association.

Previous studies have reported higher calprotectin concentrations in breastfed children compared with formula-fed children [11]. We did not find any difference in gut inflammation markers (human $\beta$ defensin-2 and calprotectin) when comparing children that were breastfed for 3 or 6 months or longer with children that were breastfed less than 3 or 6 months. It would have been interesting to analyse whether there would be differences at other age points. Unfortunately, however, data for gut inflammation marker concentrations were available only at 3 and at 6 months of age.

The possibility of finding at least some of the differences just by chance cannot be ruled out. However, whenever a statistical difference was observed in the current study, the median of the immunological marker was consistently lower in children that were breastfed for 6 months or longer compared with children that were breastfed for less than 6 months. Possible generalisability of the results to a non-risk population can, unfortunately, not be sorted out in this study, since the DIABIMMUNE study inclusion criteria included only children carrying increased genetic risk for type 1 diabetes.

Breastfeeding for 6 months or longer was associated consistently with lower medians of altogether 14 serum immunological markers at one or more time points during the first 2 years of life. At 36 months of age, no differences were seen in serum immunological markers in relation to earlier breastfeeding history. The clinical meaning of the findings is not clear, because no direct association with clinical type 1 diabetes could be determined in this study setting and because previous studies have not defined normal levels of serum immunological markers during infancy. However, the present study contributes to the understanding of immunological differences in children who have been breastfed for a longer period, and accordingly provides a potential mechanism to the association previously observed between breastfeeding and risk of type 1 diabetes.

Data availability The authors confirm that, for approved reasons, some access restrictions apply to the datasets generated during and/or analysed during the current study underlying the findings. Researchers interested in using the data are required to follow the terms of a number of clauses designed to ensure the protection of privacy and compliance with relevant regulations. Data are available upon request due to ethical restrictions, pending approval from the relevant ethical committees.

Funding Open Access funding provided by National Institute for Health and Welfare (THL). The work was supported by the European Union Seventh Framework Programme (grant no. 202063), the Academy of Finland (decision no. 292538, Centre of Excellence in Molecular Systems Immunology and Physiology Research, decision no. 250114) and the Liv och Hälsa Fund, and through an EFSD award supported by the EFSD/JDRF/Lilly.

Authors' relationships and activities The authors declare that there are no relationships or activities that might bias, or be perceived to bias, their work.

Contribution statement MEM, JH, SN, SMV and MK were responsible for conception and design of the study. JH, OV, SMV and MK were responsible for the acquisition of data. MEM analysed the data. JH and MK supervised laboratory analysis of immunological markers. All authors contributed to the interpretation of the data. MEM drafted the article with contributions from JH, SN, SMV and MK. All authors critically reviewed and approved the version to be published. MK and SMV are the guarantors of this work.

Open Access This article is licensed under a Creative Commons Attribution 4.0 International License, which permits use, sharing, adaptation, distribution and reproduction in any medium or format, as long as you give appropriate credit to the original author(s) and the source, provide a link to the Creative Commons licence, and indicate if changes were made. The images or other third party material in this article are included in the article's Creative Commons licence, unless indicated otherwise in a credit line to the material. If material is not included in the article's Creative Commons licence and your intended use is not permitted by statutory regulation or exceeds the permitted use, you will need to obtain permission directly from the copyright holder. To view a copy of this licence, visit http://creativecommons.org/licenses/by/4.0/.

\section{References}

1. Laforest-Lapointe I, Arrieta MC (2017) Patterns of early-life gut microbial colonization during human immune development: an ecological perspective. Front Immunol 8:788

2. Bach JF (2018) The hygiene hypothesis in autoimmunity: the role of pathogens and commensals. Nat Rev Immunol 18(2):105-120. https://doi.org/10.1038/nri.2017.111

3. Stewart CJ, Ajami NJ, O'Brien JL et al (2018) Temporal development of the gut microbiome in early childhood from the TEDDY 
study. Nature 562(7728):583-588. https://doi.org/10.1038/s41586018-0617-x

4. Moodley-Govender E, Mulol H, Stauber J, Manary M, Coutsoudis A (2015) Increased exclusivity of breastfeeding associated with reduced gut inflammation in infants. Breastfeed Med 10(10):488492. https://doi.org/10.1089/bfm.2015.0110

5. Lewis ED, Richard C, Larsen BM, Field CJ (2017) The importance of human milk for immunity in preterm infants. Clin Perinatol 44(1):23-47. https://doi.org/10.1016/j.clp.2016.11.008

6. Craig ME, Kim KW, Isaacs SR et al (2019) Early-life factors contributing to type 1 diabetes. Diabetologia 62:1823-1834. https://doi.org/10.1007/s00125-019-4942-x

7. Mustonen N, Siljander H, Peet A et al (2018) Early childhood infections precede development of beta-cell autoimmunity and type 1 diabetes in children with HLA-conferred disease risk. Pediatr Diabetes 19(2):293-299. https://doi.org/10.1111/pedi.12547

8. Lundberg M, Krogvold L, Kuric E, Dahl-Jorgensen K, Skog O (2016) Expression of interferon-stimulated genes in Insulitic pancreatic islets of patients recently diagnosed with type 1 diabetes. Diabetes 65:3104-3110. https://doi.org/10.2337/db16-0616

9. Kallionpaa H, Elo LL, Laajala E et al (2014) Innate immune activity is detected prior to seroconversion in children with HLA-conferred type 1 diabetes susceptibility. Diabetes 63:2402-2414. https://doi. org/10.2337/db13-1775

10. Ferreira RC, Guo H, Coulson RM et al (2014) A type I interferon transcriptional signature precedes autoimmunity in children genetically at risk for type 1 diabetes. Diabetes 63:2538-2550. https:// doi.org/10.2337/db13-1777

11. Li F, Ma J, Geng S, Wang J, Ren F, Shneg X (2014) Comparison of the different kinds of feeding on the level of fecal calprotectin. Early Hum Dev 90(9):471-475. https://doi.org/10.1016/j.earlhumdev. 2014.06.005

Publisher's note Springer Nature remains neutral with regard to jurisdictional claims in published maps and institutional affiliations. 